\title{
Urban canyon effect: storm drains enhance call characteristics of the Mientien tree frog
}

\author{
W.-H. Tan1, C.-G. Tsai², C. $\operatorname{Lin}^{1} \&$ Y. K. $\operatorname{Lin}^{1,3}$ \\ 1 Department of Life Sciences, National Taiwan University, Taipei, Taiwan \\ 2 Graduate Institute of Musicology, National Taiwan University, Taipei, Taiwan \\ 3 Institute of Ecology and Evolutionary Biology, National Taiwan University, Taipei, Taiwan
}

\begin{abstract}
Keywords
amphibian; bioacoustics; sound property; perch selection; Taiwan.

\section{Correspondence \\ Y. Kirk Lin, Department of Life Sciences, National Taiwan University, No. 1, Sec. 4, Roosevelt Rd., Taipei 10617, Taiwan. Tel: +886-2-33664534; Fax: +886-2-33662489 Email: kirklin@ntu.edu.tw}

Editor: Mark-Oliver Rödel

Received 10 October 2013; revised 28 April 2014; accepted 28 April 2014

doi:10.1111/jzo.12154

\begin{abstract}
Anthropogenic structures, such as wall surfaces, may change the acoustic environment for signals transmitted by animals, creating novel environments that animals must either adapt to or abandon. Animals can potentially use those structures to manipulate sound characteristics. In many anuran species, successful reproduction depends on long-range propagation and perception of advertisement calls. Callers may select natural perches or human-made objects to assist call propagation. Male Mientien tree frogs Kurixalus idiootocus frequently perch and call in roadside concrete drainages - miniature urban canyons. We used a combination of field and indoor experiments to test two hypotheses: (1) transmission of calls emitted inside drains is enhanced; (2) males selected perches inside drains that facilitated call transmission. A field survey indicated that male Mientien tree frogs preferred calling inside rather than outside drains. A playback showed that calls emitted from inside drains were enhanced in both amplitude and note duration. In an indoor experiment using a replica of a concrete drain, males preferred one particular type of call perch. However, we found no difference in sound properties between random locations inside the drain model and the perch location preferred by calling males.
\end{abstract}

\section{Introduction}

Acoustic signals play fundamental roles in anuran communication by facilitating social interactions, mediating reproduction and determining vulnerability to predation (reviewed in Ziegler, Arim \& Narins, 2011). In many species, successful reproduction depends on the long-range propagation and perception of advertisement calls (Kuczynski et al., 2010). Auditory signals, however, may not be able to travel over long distances because intensity may attenuate and structure may degrade (Naguib \& Wiley, 2001; Wells, 2007). Many animals have evolved strategies to sustain long-distance communication (Slabbekoorn \& Smith, 2002; Bosch \& de la Riva, 2004; Boncoraglio \& Saino, 2006). As in other taxonomic groups (e.g. birds, Barker \& Mennill, 2009), several anuran species have been shown to explore habitat features that assist call propagation (Lardner \& Lakim, 2002; Cui, Tang \& Narins, 2012). For example, Lardner \& Lakim (2002) demonstrated that tree-hole frogs Metaphrynella sundana exploited resonance effects of tree-holes to enhance call propagation, thus affecting female choice (Lardner \& Lakim, 2004). Anurans may also alter perceived call quality by transforming habitat features. Cui et al. (2012) found that male Emei music frogs Babina dauchina constructed nests that changed signal pro- perties. Males' calls faithfully advertised whether or not they possessed a nest to potential mates.

Anthropogenic structures, such as wall surfaces, may change the acoustic environment for signals transmitted by animals (Kight, Hinders \& Swaddle, 2012), creating novel environments that animals must either adapt to or abandon (Warren et al., 2006). Animals can potentially use those structures to manipulate sound characteristics, but no relevant empirical study has yet emerged. In Taiwan, open concrete drainages are a common feature in suburban and rural areas. These drains - miniature urban canyons - are usually built alongside paved roads or foot trails. Depending on the slope of the drains and surrounding environments, the floors can be thick with mud and plant litter. Many frogs and invertebrate species inhabit, and sometimes reproduce, in these drains. Some animals use them as movement corridors. The impervious parallel concrete walls allow sounds to ricochet and linger, and can alter sound characteristics due to reverberation (Slabbekoorn, Ellers \& Smith, 2002; Warren et al., 2006; Kight et al., 2012). Particularly, animal signals produced in drains may be degraded by echo. Receivers will hear not just the direct sound wave, but also reflected waves arriving at different times (Warren et al., 2006). In contrast, some signals (e.g. those with narrow frequency bandwidths) can potentially 
benefit from reverberations. The reflected sound waves can make the signal higher in amplitude and longer (Slabbekoorn et al., 2002).

In this study, we investigated whether concrete storm drains altered acoustic characteristics of the Mientien tree frog Kurixalus idiootocus. This frog is a common endemic species in Taiwan. With a dominant frequency of $2-3 \mathrm{kHz}$ (Zheng, 1989), the male advertisement call is more high-pitched than most of other frogs native to Taiwan. Males are often observed perching and calling in roadside drains; females have been observed coming to drains or perching on nearby vegetation. We asked two research questions. First, does calling from drains versus non-drain habitats produce sounds with different acoustic characteristics? We tested the hypothesis that sounds emitted from drains are louder than non-drain habitats and that males preferentially select drains as calling sites. Since different locations within a drain may have different acoustic properties, we asked a second question: do males select particular calling perches that enhance call characteristics? We tested the hypothesis that sounds emitted from frog-selected perches are louder than random locations within drains.

\section{Methods}

\section{Study site}

The study site was located in a suburban woodland ( $25^{\circ} 01^{\prime}$ 02.97", E $\left.121^{\circ} 33^{\prime} 14.44^{\prime \prime}\right)$ in south-eastern Taipei, Taiwan.
Paved roads crisscross the site. Open concrete drains are on one or both sides of each road. Many male Mientien tree frogs perch inside these drains or on nearby vegetation, forming choruses during the breeding season, from February to September.

\section{Measuring calls}

We obtained recordings of advertisement calls from several male Mientien tree frogs at similar distances, and examined the acoustic waveform and spectrogram of 20 recordings. We chose one call that had characteristics representative of the population and little background noise as the standard call for subsequent playback experiments. The standard call contained 13 notes with a dominant frequency at $2 \mathrm{kHz}$ (Fig. 1). The sound pressure level rose in the first 11 notes. We examined five call variables in both field and laboratory experiments: average power (average sound pressure level in $\mathrm{dB}$ ), maximum power (maximum sound pressure level in $\mathrm{dB}$ ), sum note duration (sum of duration of all notes in a call, excluding the intervals of silence between notes), rising time ratio (ratio in time between rising sound pressure period and total call period) and dominant frequency (frequency in $\mathrm{kHz}$ at which maximum sound pressure level occurs). All five variables were calculated based on the spectrograms of calls. For example, we calculated the average power by averaging the power spectral density over the frequency-time range that we selected on the spectrogram.

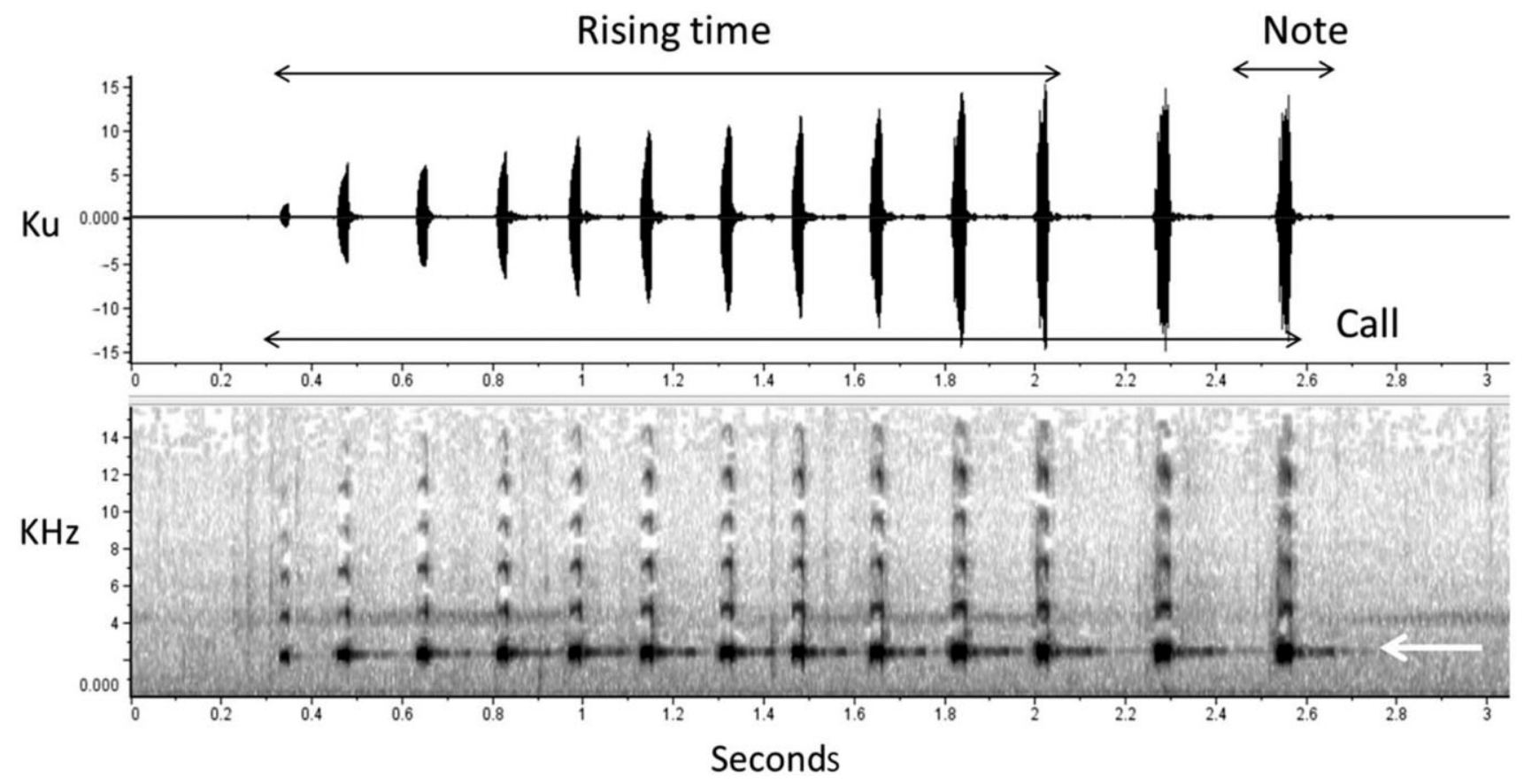

Figure 1 Characteristics of the Mientien tree frog Kurixalus idiootocus advertisement call. This 13-note call was used in both field and laboratory playback experiments. White arrow indicates dominant frequency: $2-3 \mathrm{kHz}$. Rising time is the time length during which sound pressure level rises. 


\section{Field survey and playback experiment}

We established 11 randomly selected plots along a trail at the study site to survey the number of frogs. Each plot was $10-\mathrm{m}$ long. We delineated the 0.5 -m-wide drain as drain habitat and the 10-m-wide land beside the drain as upland habitat. After dark, we approached each plot quietly and counted the number of Mientien tree frogs in each habitat. Each plot was surveyed twice: on a rainy day and a non-rainy day, separated by 10 days. The vegetation coverage by the drain and mudand-litter thickness in the drain in each plot were recorded.

We chose a plot (playback plot, hereafter) that was representative of the survey plots to conduct a perch location survey and playback experiment. There was a chorus of frogs at this site throughout the breeding season. For 5 nonconsecutive evenings, we visited the playback plot and recorded perch locations of calling Mientien tree frogs in the drain and upland habitats. We selected 15 drain and 15 upland perch locations, at which we conducted a playback experiment from 18:00 to 23:00 h on an August evening to examine effects of habitat (drain vs. upland) on call transmission. Note that the perch, thus playback, locations varied in heights. Playback was broadcast from an audio player (Soundlook, SDD-5000, Koizumi Seiki Corporation, Tokyo, Japan) with an external speaker (Lifetrons, LT-8006, Lifetrons Switzerland; Hong Kong, China) at each location. Depending on the perch heights, the speaker was placed at different elevations, facing upward. The broadcast lasted 30-60 seconds to allow recording of at least 10 calls with good quality. We recorded the playback with a directional microphone (Sony, ECM-CG50, Sony Corporation, Tokyo, Japan) attached to a recorder (Sony, PCM-M10, Sony Corporation). The microphone was placed at $70 \mathrm{~cm}$ directly above and facing the speaker. Because the playbacks did not involve live animals, we were able to keep a precise distance between the microphone and speaker. We used this recording configuration for two reasons. First, recording unobstructed sounds from multiple directions at multiple fixed distances was not possible without disturbing surrounding vegetation. Second, female tree frogs often perch on overhead vegetation to listen to the male chorus (Zheng, 1989); therefore, males must transmit their calls upward to attract females.

\section{Indoor perch selection and playback experiment}

In an empty room, we constructed a concrete drain (length: $300 \mathrm{~cm}$; width: $40 \mathrm{~cm}$; height: $60 \mathrm{~cm}$ ) that mimicked drains in the field. Concrete slabs (length: $150 \mathrm{~cm}$; thickness: $3 \mathrm{~cm}$; height: $30 \mathrm{~cm}$ ) supported from outside by a steel frame formed parallel walls standing on a concrete floor. Slabs had been weathered and dampened outdoors for 2 weeks before construction. The two open ends of the drain were blocked by soundproofing polyester foam (5-cm-thick) glued on a styrofoam board (3-cm-thick) to absorb sounds transmitted toward the ends. We covered the drain floor with a 5-cm layer of damp soil and a thin layer of leaf litter. We laid four water dishes (length: $11 \mathrm{~cm}$; width: $7 \mathrm{~cm}$; depth: $4 \mathrm{~cm}$ ) and four boulders (about $10-\mathrm{cm}$ diameter), equally spaced, on the floor. Onethird and two-thirds of the way along the drain, we leaned two tree branches (65-cm length; 5-cm diameter) diagonally across walls in the drain. The drain offered perch surface on the floor, walls and branches. Finally, we secured the open-topped drain with plastic window screens, which had minimal effect on sound transmission, to prevent frogs from escaping. The drain was located in the center of a room with curtain-covered walls. The ceiling was made of sound-absorbing materials.

On a testing evening, we collected three male Mientien tree frogs from the study site and selected the male with the highest calling activity for the perch selection experiment (see Ethical notes for details). We placed him on a random location inside the drain. A recording of the Mientien tree frog chorus (recorded at the field study site) was broadcast in the background to imitate field conditions and to stimulate calling. An audio recorder was hung above the drain to record the male's vocal response. We left the tested male alone for an hour. Then, we entered the room quietly to record his perch location three times, separated by 20 -min intervals. Overall, the trial lasted $100 \mathrm{~min}$. Each tested male would yield three perch locations. However, only the location where a male was actually calling would be considered a calling perch. Males with 0 calling perches were excluded from analysis. For those that had more than one calling perch, we used the first perch location for the subsequent analysis. A perch location was recorded by superimposing a two-dimensional coordinate system with a $5-\mathrm{cm}$ resolution on the available perch surface on the drain floor and walls and branches. Although the branches are threedimensional, frogs only perch on the upper hemisphere. We consider the thickness of the branch negligible. We classified perch locations into three categories: on branch, floor or wall. Total available perches for each category were quantified by number of $5 \times 5$-cm areas that could be occupied by frogs. We tested a total of 20 males. Five did not call during any intervals of a trial and were excluded from subsequent analyses. To compare with the 15 frog-selected locations, we generated 15 randomly selected locations by Matlab v7.7 (The MathWorks Inc, Natick, MA, USA). We then conducted a playback experiment to examine effects of perch selection on call transmission. Playback devices and procedures were similar to those used in the field experiment, except that we recorded broadcast $3 \mathrm{~cm}$ above the speaker. Because the playbacks did not involve live animals, we were able to keep a precise distance between the microphone and speaker.

\section{Ethical notes}

We captured three males by hand per test night and placed them in individual plastic amphibian holding tanks (length: $15 \mathrm{~cm}$; width: $11 \mathrm{~cm}$; height: $7 \mathrm{~cm}$ ) supplied with leaf litter and wet tissue towel. We were able to capture all three frogs in less than $30 \mathrm{~min}$ and to transport them back to laboratory in $20 \mathrm{~min}$. Upon returning to the laboratory, the three males were immediately housed in a glass tank (length: $45 \mathrm{~cm}$; width: $29 \mathrm{~cm}$; height: $27 \mathrm{~cm}$ ) with window screen top in room temperature. There was no air conditioning. Since the experiment was conducted in August, room temperature ranged from 25 
to $28^{\circ} \mathrm{C}$, and was $1-2^{\circ} \mathrm{C}$ warmer than field temperature. The tank floor was moist sphagnum moss covered with leaf litter and supplied with a shelter made of flowerpot. We provided water and food (fruit flies and meal worms) ad lib. The glass housing tank followed the light:dark cycle of the laboratory light on from 08:00 to 19:00 $\mathrm{h}$. The frogs usually started calling shortly after we placed them in the glass tank.

The perch selection experiment started as soon as the frogs were calling actively. We chose the individual with the greatest calling activity for the experiment. Tested males were returned to the glass tank immediately after trials. All males were released at the site of capture within 3 days following the experiment. We did not see any apparent effects on health or mortality during transportation, experiment and laboratory housing. Working with wild animals with no conservation concern and without long-term housing does not require a permit from our institute or government. The procedures of the study followed the ASAB/ABS Guidelines for the Use of Animals in Research.

\section{Acoustic analysis}

We first processed all 60 recordings (sampled at $44.1 \mathrm{kHz}$ and quantized at 16 bits), 30 from the field and 30 from the indoor experiments, with Adobe Audition v1.5 (Adobe Systems Inc., San Jose, CA, USA). The microphone of the recorder we used has two recording channels. We examined and found the soundtracks recorded by the two channels were nearly identical in all acoustic characteristics. To simplify the subsequent analyses, for each recording, we combined both soundtracks into one by averaging values of the two. Next, we analyzed calls with Raven Pro 1.4 (Bioacoustics Research Program, Cornell Lab of Ornithology). We used the band limited energy detector function to help identify the boundaries of individual calls in each recording. Since calls were playbacks of the standard call, we did not expect substantial variations in playback at the same location. We therefore randomly sampled three calls from each recording and calculated means for the measured variables. Analyses were based on spectrograms generated by short-time Fourier transform (STFT) (Hann window, sampling rate $44100 \mathrm{~Hz}$, FFT size 1024, step size 512) in Raven Pro (v. 1.4).

\section{Statistical analyses}

Field and laboratory datasets were examined by Shapiro tests for normality and by $F$-tests for variance homogeneity assumptions before parametric statistical tests. We used equivalent non-parametric tests when a dataset failed one or both assumptions (see below). Densities of calling Mientien tree frogs from the two surveys were averaged before comparing differences between drain and upland habitats with a paired $t$-test. Overall differences in call variables between drain and upland recordings were analyzed by Hotelling's $\mathrm{T}$-square test. We compared individual variables with twosample $t$-tests (or Mann-Whitney tests) with Bonferroni corrections. Perch selection by males within laboratory drain was analyzed by a chi-square test. We did all statistical analyses using SAS 9.2 (SAS Institute, Cary, NC, USA) and report mean \pm 1 standard error.

\section{Results}

\section{Field survey and playback experiment}

There was a greater density of calling males inside the drain $\left(1.64 \pm 0.40\right.$ males per $\left.\mathrm{m}^{2}\right)$ than in upland habitat $(0.02 \pm 0.01$ males per $\mathrm{m}^{2}$; paired $t$-test: $\left.t_{10}=4.08, P<0.002\right)$. There was a significant overall difference across all five call variables between calls broadcast from inside the drain versus those from upland habitat (Hotelling's T-square test: $F_{5,24}=3.30$, $P=0.02$ ). Examining call variables separately (alpha-adjusted to 0.01 with Bonferroni corrections) revealed significant differences (Fig. 2) in average power (two-sample $t$-test: $t_{28}=3.77, P<0.001$ ), maximum power (two-sample $t$-test: $t_{28}=3.84, P=0.0006$ ) and sum note duration (two-sample $t$-test: $\left.t_{28}=3.08, P=0.005\right)$, but not in rising time ratio (Mann-Whitney test: $U=0.25$, d.f. $=1, P=0.80$ ) and dominant frequency (Mann-Whitney test: $U=0.92$, d.f. $=1$, $P=0.36)$. Generally, sounds transmitted from drains had greater values than those from upland habitats.

\section{Laboratory experiment}

Mientien tree frogs did not select perch locations proportionally to their availabilities (chi-square test: $\chi^{2}=286.33$, $P<0.0001$ ). Selections by the 15 frogs were 9,3 and 3 on branch, floor and wall, respectively, compared with an availability of 18, 400 and 578, respectively. Males clearly preferred leaning tree branches and avoided drain walls and floor.

There was no significant overall difference in all five call variables between calls broadcast from random and frogselected locations (Hotelling's T-square test, $F_{5,24}=0.11$, $P=0.99$ ). Examining each call variable separately (alphaadjusted to 0.01) did not reveal any significant differences (Fig. 3): average power (Mann-Whitney test, $U_{15,15}=0.08$, $P=0.93$ ), maximum power (Mann-Whitney test, $U_{15}$, $15=0.29, P=0.77$ ), sum note duration (Mann-Whitney test, $U_{15,15}=-0.19, P=0.85$ ), rising time ratio (Mann-Whitney test, $U_{15}, 15=0.17, P=0.87$ ) and dominant frequency (MannWhitney test, $U_{15,15}=0.34, P=0.73$ ).

\section{Discussion}

Studies examining human effects on bioacoustics tend to focus on the consequences of anthropogenic noise on communication. This study reveals a novel effect - human-made drainage ditches alter the characteristics of frog calls. The field playback experiment showed that frog calls emitted from inside drains had greater intensity of sound (in both average and maximum power) and longer duration (sum note duration) than calls produced in upland habitats (Fig. 2). These calls may exert a higher attraction on females. Numerous studies on amphibians have shown that females were attracted to 

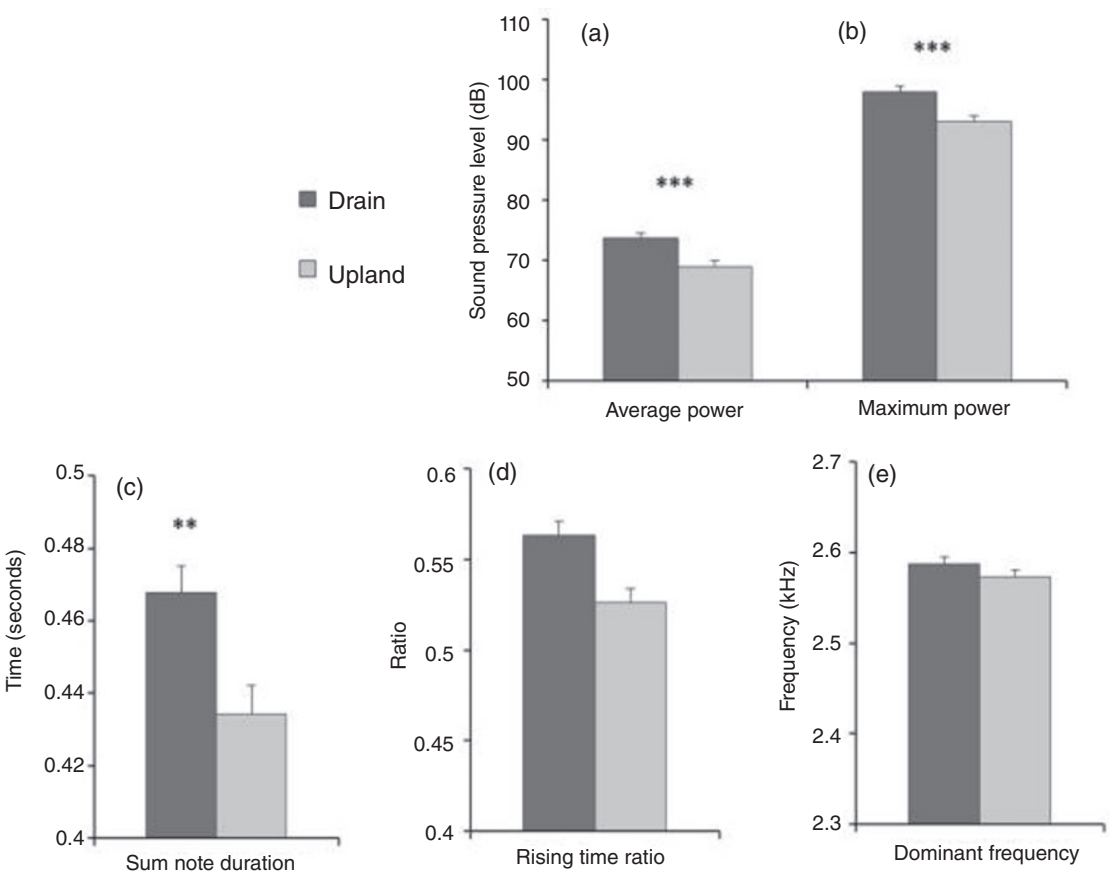
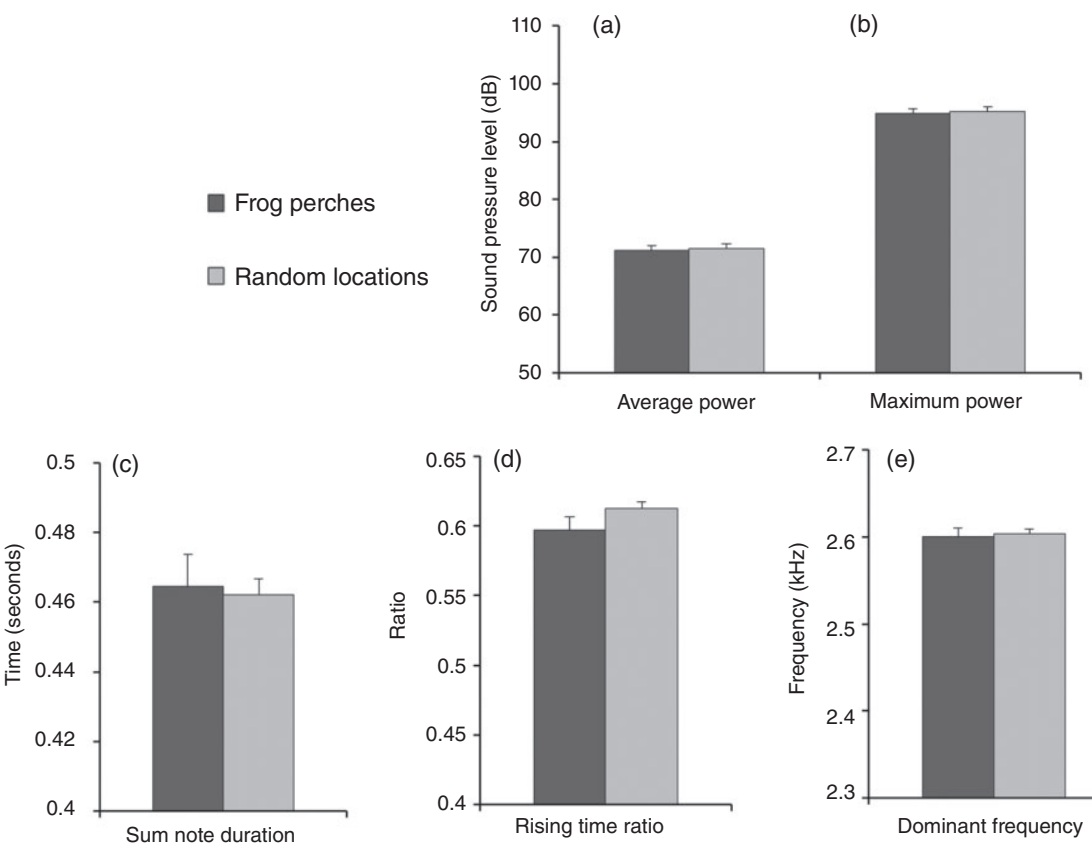

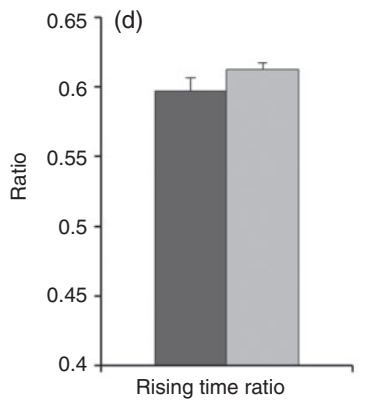

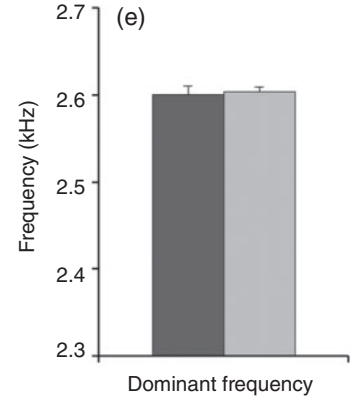

Figure 2 Five properties of a standardized playback call when broadcasted from inside a drain versus from upland (i.e. far outside drain) habitats: (a) average power, (b) maximum power, (c) sum note duration, (d) rising time ratio and (e) dominant frequency. Asterisks above bars indicate significant differences $\left({ }^{*} P<\right.$ $\left.0.01,{ }^{*}{ }^{*} P<0.001\right)$.

Figure 3 Five properties of a standardized playback call when broadcasted from Mientien tree frog Kurixalus idiootocus perches versus from random locations in a storm drain replica: (a) average power, (b) maximum power, (c) sum note duration, (d) rising time ratio and (e) dominant frequency. There was no significant difference between calls recorded at frog perches versus random locations in any sound properties.

male calls with long call duration (Gerhardt, 1991; Gerhardt et al., 2000; Smith \& Roberts, 2003) and high amplitude (Gerhardt, 1987; Ryan \& Rand, 1990; Forrest \& Raspet, 1994).

The impervious parallel concrete walls of drains could produce reverberation of male calls (Slabbekoorn et al., 2002; Warren et al., 2006; Kight et al., 2012). We did not detect calls being degraded by echo (Warren et al., 2006). Instead, the calls became louder and longer than those on the upland. This was likely caused by combined effects of drain dimension (Warren et al., 2006) and frog call frequency bandwidth (Slabbekoorn et al., 2002). Warren et al. (2006) showed that sounds emitted in narrow canyons had longer signal decay times, thus could maintain amplitude for longer distance. 
Narrow canyons may also cause greater sound distortion due to the many reflected waves arriving at different times. However, Slabbekoorn et al. (2002) pointed out that sound signals with narrow frequency bandwidths (pure tones) would be less distorted by reverberations. The reflected sound waves can make a pure tone higher in amplitude and longer (Warren et al., 2006). Mientien tree frog calls, with a narrow frequency bandwidth, may benefit from calling in drainage ditches. Furthermore, a field survey found that more male Mientien tree frogs called from drains than upland habitats. This suggested that the species may be actively exploiting the acoustic effects offered by drains.

Males that call with better sound transmission are expected to be favored by sexual selection (Lardner \& Lakim, 2004; Cui et al., 2012). However, high-frequency advertisement calls, as in Mientien tree frogs, are easily attenuated with distance and degraded by obstacles (Narins, 2007). Thus, for a given sound volume, high-frequency callers face a trade-off in sound propagation. They need to choose between calling toward a particular direction for a distant selected audience or toward all directions to reach a nearby broad audience. Increasing sound volume can alleviate such a tradeoff (Narins, 2007). Advertisement calls of Mientien tree frogs are dominated by high-frequency signals $(2-3 \mathrm{kHz})$. We do not know if the species could broadcast calls toward a particular direction. The calls of some species (e.g. Hyla cinerea, Gerhardt 1975) are known to be omnidirectional. Thus, Mientien tree frogs may not be able to send calls toward a particular direction for distant audience to make up for attenuation and degradation. Our results showed that calling in the drains can help alleviate the problem of signal attenuation and degradation because drains can enhance sound power and signal duration.

Drainage ditches are linear structures. Sounds could travel strongly forward, backward and also upward. Although the upward direction may be beneficial because female Mientien tree frogs were often observed perching on trees or vegetation (Zheng, 1989), the drain walls could obstruct call transmission toward receivers, including approaching females at ground level. Such a potential disadvantage could be lessened by calling from perches close to the top of drain walls. Indeed, we observed some calling males do so in the field and laboratory. Unfortunately, we could not examine such effects on transmission since we did not record male calls from difference directions or elevations from sound source.

The mating system of the Mientien tree frog has been classified as a lek mating system (Zheng, 1989). Zheng (1989) investigated the reproductive behaviors of the Mientien tree frog. He found that the species usually formed choruses in large aggregations and females would visit aggregations. Our field survey found males distributed in discrete areas. Some areas were dense with calling males, while nearby areas may have none. For example, on an evening in May, the number of calling males within survey plots (10-m sections of drain and upland) ranged between 0 and 15 . Fifty per cent of them had 0-3 males, while only $14 \%$ had 12 or more males. According to Zheng (1989), after a female meets with her choice of male, the female will carry the male to damp loca- tions, often near shores of still water, and deposit eggs among leaf litter or in soil crevices. Indeed, we observed that the sections of drain where large aggregations of males occur did not necessarily provide breeding substrates (mud and leaf litter). In fact, we are not sure where Mientien tree frogs laid their eggs in our study site. Although we observed a lot of males and some females in the drains during the breeding seasons, we only found very few egg clusters in the drain, and even fewer were at lek sites.

In a lek mating system, females attend the lek (chorus) before selecting an individual as mate. As a result, joining a high-quality lek is critical for the reproductive success of males (Bee, 2007). Assuming that females are attracted to leks with intensely calling males, then males calling from drains may benefit because their calls are intensified by the drains. Although we cannot be sure that acoustic property is the sole reason that males chose drains for calling, it seems that resources (e.g. breeding substrates) may not be an important factor in their mate choice. Furthermore, the species is a tree frog, which has high desiccation tolerance. Thus, males may not face strong physiological pressure to choose drains for maintaining water balance. Taken together, we think that acoustic advantage largely accounts for the observation that males preferred to call in the drains. Nevertheless, drain habitats pose substantial challenges for calling males and visiting females. For example, it may be physically demanding for females to carry males up vertical walls. Thus, drains could be ecological traps if they in fact reduce reproductive success of individuals attending choruses in drains.

The indoor perch selection experiment showed that male Mientien tree frogs prefer to call from branches, which had low availability, inside drains. However, we did not find that calling from selected perch locations changed any acoustic characteristics of calls we examined. These contradictory results might indicate that our playback recording procedure was inadequate. We used a $3-\mathrm{cm}$ recording distance to examine the effects because a calling male likely listened to its own calls and moved to locations where it could hear itself best. It should also be where its calls would be transmitted the best. Nevertheless, the sound waves travelled merely $3 \mathrm{~cm}$ directly from speaker to microphone, and their strong energy may have overshadowed any sound reflections from the walls in the recordings. Alternatively, preference for leaning branches may be due to other considerations: improved view of approaching females, competing males and predators. Compared with upland habitat, concrete drains offer a view with little obstruction. In fact, we often observed snakes, particularly Chinese green tree viper Trimeresurus stejnegeri in these drains. Concrete walls seem to provide the benefit of enhancing thermal regulation, consequently become a preferred ambushing location for vipers (J. J. Mao, pers. comm.). Perching on branches would not only improve visibility, but also increase the chances of escaping predator attacks. In songbirds, variation in singing perch height reflects a trade-off among ideal locations for different activities, such as singing, nest-building, foraging and mate guarding (Barker \& Mennill, 2009). Therefore, we propose that male Mientien tree frogs choose to call in drains because of enhanced transmission, 
but selection for perch location inside drains is determined by multiple factors. Sound transmission may not play a dominant role here.

Concrete drains are structures built by humans for storm management, a structure new to frogs in evolutionary time. Many anthropogenic structures have characteristics similar to those in natural habitats. Skyscraper ledges are used as perches and nesting sites by cliff-living birds, and building crevices are used by many cavity-nesting birds. Warren et al. (2006) suggested that the common 'two parallel hard wall' feature of urban canyons in the cities may affect acoustic characteristics of and are explored by city-dwelling animals. As far as we know, no study has examined the use of anthropogenic structure by wildlife to manipulate vocal communication (Ortega, 2012). Concrete drains are miniature canyons, but are not analogous to anything in Mientien tree frog natural habitats. Therefore, it is interesting to find those frogs preferentially calling in the drains. Mientien tree frogs, with their high-frequency calls, should benefit from using drains, which help sustain amplitude over long distances. The mechanism by which frogs selected perches that could enhance sound transmission was not clear. A male may simply move to locations with the loudest callers. A male may also listen to his own calls, moving to where his calls are transmitted the best.

Finally, we recognize that we should have used more than one call exemplar in the field and indoor playback experiments. Using a single naturally recorded call makes it difficult to generalize results to the species as a whole because there can be unique properties of the chosen call that may have led to the results obtained in this study. As mentioned earlier, we chose the exemplar because it reflected the average of the population we recorded. Thus, our results should faithfully reflect the expected sound transmission for an average male under these conditions. In addition, this study did not use the playback to elicit responses of live frogs. The responses were all from physical properties of drains in the field and laboratory. In summary, this study addresses a novel consequence of anthropogenic influences on natural populations. Humanmade storm drains influence the acoustic properties of frog calls, and males in fact prefer to call in drains. Although calling males in drains seemingly gain acoustic advantages, other negative consequences of calling in drains remain to be explored in order to determine whether such behavior ultimately increases or reduces fitness.

\section{Acknowledgments}

We thank Shu-Hui Kuan, Jo-Hua Liang, Cheng-Hao Lin, Wei-Son Lien, Chung-Hao Juan, Wen-Chi Lin, Yi-Hsun Tan, Li-Wen Chang, Shan-Ta Tao, Bei-Shan Lee, Yi-Lun Lin, Po-Han Chou, Eileen Lee, Yu-San Yang, Chia-Chien Lee, Po-Ting Yeh, Chia-Che Lin, Che-Hao Chen, Ching-Ho Hou and Yu-Hui Lin for their help in the field. We thank Yi-Lun Lin, Bei Shan Lee, Shan-Ta Tao, Wei-Ting Yeh, Ching-Ho Hou and Po-Han Chou for assistance in building the indoor drain. We thank Po-Ting Yeh and Chun-Yen Huang for constructive comments on acoustics related to this study. We thank Dr Cara Lin Bridgman for improving the structure and language of the paper. We thank the three anonymous reviewers for their constructive comments on earlier versions of the paper. We thank National Science Council for the College Student Research Scholarship (NSC 101-2815-C-002-161-B) to Wen-Hao Tan and a research grant (NSC 98-2621-B-002005-MY3) to Y. Kirk Lin.

\section{References}

Barker, N.K.S. \& Mennill, D.J. (2009). Song perch height in rufous-and-white wrens: does behaviour enhance effective communication in a tropical forest? Ethology 115, 897-904.

Bee, M.A. (2007). Selective phonotaxis by male wood frogs (Rana sylvatica) to the sound of a chorus. Behav. Ecol. Sociobiol. 61, 955-966.

Boncoraglio, G. \& Saino, N. (2006). Habitat structure and the evolution of bird song: a meta-analysis of the evidence for the acoustic adaptation hypothesis. Functional Ecology 21, 134-142.

Bosch, J. \& de la Riva, I. (2004). Are frog calls modulated by the environment? An analysis with anuran species from Bolivia. Can. J. Zool. 82, 880-888.

Cui, J., Tang, Y. \& Narins, P.M. (2012). Real estate ads in Emei music frog vocalizations: female preference for calls emanating from burrows. Biol. Lett. 8, 337-340.

Forrest, T. \& Raspet, R. (1994). Models of female choice in acoustic communication. Behav. Ecol. 5, 293-303.

Gerhardt, H.C. (1975). Sound pressure levels and radiation patterns of the vocalizations of some North American frogs and toads. J. Compar. Physiol. 102, 1-12.

Gerhardt, H.C. (1987). Evolutionary and neurobiological implications of selective phonotaxis in the green treefrog, Hyla cinerea. Anim. Behav. 35, 1479-1489.

Gerhardt, H.C. (1991). Female mate choice in treefrogs: static and dynamic acoustic criteria. Anim. Behav. 42, 615-635.

Gerhardt, H.C., Tanner, S.D., Corrigan, C.M. \& Walton, H.C. (2000). Female preference functions based on call duration in the gray tree frog (Hyla versicolor). Behav. Ecol. 11, 663-669.

Kight, C.R., Hinders, M.K. \& Swaddle, J.P. (2012). Acoustic space is affected by anthropogenic habitat features: implications for avian vocal communication. Ornithological Monographs 74, 47-62.

Kuczynski, M.C., Vélez, A., Schwartz, J.J. \& Bee, M.A. (2010). Sound transmission and the recognition of temporally degraded sexual advertisement signals in Cope's gray treefrog (Hyla chrysoscelis). J. Exp. Biol. 213, 2840-2850.

Lardner, B. \& Lakim, M.B. (2002). Tree-hole frogs exploit resonance effects. Nature 420, 475.

Lardner, B. \& Lakim, M.B. (2004). Female call preferences in tree-hole frogs: why are there so many unattractive males? Anim. Behav. 68, 265-272. 
Naguib, M. \& Wiley, R. (2001). Estimating the distance to a source of sound: mechanisms and adaptations for longrange communication. Anim. Behav. 62, 825-837.

Narins, P.M. (2007). Hearing and sound communication in amphibians. New York: Springer Verlag.

Ortega, C.P. (2012). Effects of noise pollution on birds: a brief review of our knowledge. Ornithological Monographs 74, 6-22.

Ryan, M.J. \& Rand, A.S. (1990). The sensory basis of sexual selection for complex calls in the túngara frog, Physalaemus pustulosus (sexual selection for sensory exploitation). Evolution 44, 305-314.

Slabbekoorn, H. \& Smith, T.B. (2002). Habitat-dependent song divergence in the little greenbul: an analysis of environmental selection pressures on acoustic signals. Evolution 56, 1849-1858.

Slabbekoorn, H., Ellers, J. \& Smith, T.B. (2002). Bird-song and sound transmission: the benefits of reverberations. Condor 104, 564-573.
Smith, M.J. \& Roberts, J.D. (2003). Call structure may affect male mating success in the quacking frog Crinia georgiana (Anura: Myobatrachidae). Behav. Ecol. Sociobiol. 53, 221-226.

Warren, P.S., Katti, M., Ermann, M. \& Brazel, A. (2006). Urban bioacoustics: it's not just noise. Anim. Behav. 71, 491-502.

Wells, K.D. (2007). The ecology \& behavior of amphibians. Chicago: University of Chicago Press.

Zheng, Y.-W. (1989). The reproductive behavior of Chirixalus idiootocus. Master's thesis, National Taiwan University. [in Chinese.].

Ziegler, L., Arim, M. \& Narins, P.M. (2011). Linking amphibian call structure to the environment: the interplay between phenotypic flexibility and individual attributes. Behav. Ecol. 22, 520-526. 